PROCEEDINGS OF THE

AMERICAN MATHEMATICAL SOCIETY

Volume 129, Number 8, Pages 2319-2329

S 0002-9939(00)05806-8

Article electronically published on December 28, 2000

\title{
ALMOST PERIODIC ULTRADISTRIBUTIONS OF BEURLING AND OF ROUMIEU TYPE
}

\author{
M. C. GÓMEZ-COLLADO
}

(Communicated by Dale Alspach)

\begin{abstract}
We characterize the almost periodic ultradistributions of Beurling and of Roumieu type in terms of classical Bohr almost periodicity. Then we study the Fourier series associated with such an ultradistribution.
\end{abstract}

\section{INTRODUCTION AND NOTATION}

An almost periodic function is a function which can be uniformly approximated by trigonometric polynomials. The wide range of applications of the theory of almost periodic functions (cf. [1]), for instance to the theory of functions of a complex variable and to the theory of ordinary and partial differential equations, lead Schwartz 14 to study the almost periodicity in the setting of classical distributions. More recently almost periodic ultradistributions of Beurling type were studied by Cioranescu [5, who characterized them in terms of classical Bohr almost periodicity. She worked in the context of ultradistributions as they were introduced by Komatsu 8]. For technical reasons she required the strong non-quasianalytic condition on the defining sequence (the so-called (M3) condition). In this paper we study almost periodic ultradistributions of Beurling and of Roumieu type in the sense of Björk [2] and Braun, Meise, Taylor [4]. Recent results of Braun [3] and Langenbruch [9] concerning the existence of elliptic ultradifferential operators allow us to give in Theorem 4.2 characterizations including those of Cioranescu; moreover we also characterize the almost periodic ultradistributions of Roumieu type, a case which was not covered in [5].

One of the most important properties of the almost periodic functions is the possibility to associate to each such function a Fourier series which completely determines the function. In the last section of the paper we restrict our attention to the one variable case and we introduce the Fourier series of an almost periodic ultradistribution following the approach of Schwartz. Then we extend the FéjerBochner summation method, which permits us to construct an almost periodic ultradistribution starting with the Fourier series associated to it. This is Theorem 4.6 .

Received by the editors August 17, 1999 and, in revised form, November 30, 1999.

2000 Mathematics Subject Classification. Primary 46F05.

Key words and phrases. Bounded ultradistributions, almost periodic ultradistributions.

The author thanks C. Fernández and A. Galbis for guidance and encouragement during the preparation of her thesis, of which this work forms a part. 
We introduce the spaces of functions and ultradistributions and most of the notation that will be used in the sequel.

Definition 1.1. Let $\omega:[0, \infty[\longrightarrow[0, \infty[$ be a non-decreasing continuous function. $\omega$ is called a (non-quasianalytic) weight if it satisfies:

$(\alpha)$ there exists $L \geq 0$ with $\omega(2 t) \leq L(\omega(t)+1)$ for all $t \geq 0$,

(及) $\int_{1}^{\infty} \frac{\omega(t)}{t^{2}} d t<\infty$,

$(\gamma) \log (t)=o(\omega(t))$ as $t$ tends to $\infty$,

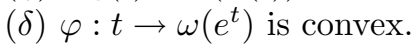

A strong weight is a weight $\omega$ satisfying the following additional condition:

$(\epsilon)$ there exists $C \geq 1$ such that for all $y>0: \quad \int_{1}^{\infty} \frac{\omega(y t)}{t^{2}} d t \leq C \omega(y)+C$.

The Young conjugate $\varphi^{*}:[0, \infty[\longrightarrow \mathbb{R}$ of $\varphi$ is defined by

$$
\varphi^{*}(s):=\sup \{s t-\varphi(t): t \geq 0\} .
$$

There is no loss of generality in assuming that $\omega$ vanishes on $[0,1]$. Then $\varphi^{*}$ has only non-negative values and $\varphi^{* *}=\varphi$.

Definition $1.2(4])$. Let $\omega$ be a weight function. For a compact set $K \subset \mathbb{R}^{N}$ and $\lambda>0$ we let

$$
\mathcal{E}_{\omega}(K, \lambda):=\left\{f \in C^{\infty}(K):\|f\|_{K, \lambda}<\infty\right\},
$$

where $\|f\|_{K, \lambda}:=\sup _{x \in K^{\prime}} \sup _{\alpha \in \mathbb{N}_{0}^{N}}\left|f^{(\alpha)}(x)\right| \exp \left(-\lambda \varphi^{*}\left(\frac{|\alpha|}{\lambda}\right)\right)$.

We define $\mathcal{E}_{(\omega)}\left(\mathbb{R}^{N}\right)$ as the set of all $C^{\infty}$ functions $f$ such that $\|f\|_{K, m}<\infty$ for each $K \subset \subset \mathbb{R}^{N}$ and each $m \in \mathbb{N}$, and $\mathcal{E}_{\{\omega\}}\left(\mathbb{R}^{N}\right)$ as the set of all $C^{\infty}$ functions such that for each $K \subset \subset \mathbb{R}^{N}$ there is $m \in \mathbb{N}$ with $\|f\|_{K, \frac{1}{m}}<\infty$.

The topology of $\mathcal{E}_{\{\omega\}}\left(\mathbb{R}^{N}\right)$ is given by first taking the inductive limit over all $m \in \mathbb{N}$ for each compact $K_{n}$ and then taking the projective limit of these, while $\mathcal{E}_{(\omega)}\left(\mathbb{R}^{N}\right)$ carries the locally convex topology given by the seminorms $\|f\|_{K_{n}, n}$, $n \in \mathbb{N}$, where $K_{n}$ is a fundamental sequence of compact subsets in $\mathbb{R}^{N}$.

The elements of $\mathcal{E}_{(\omega)}\left(\mathbb{R}^{N}\right)$ (resp. $\mathcal{E}_{\{\omega\}}\left(\mathbb{R}^{N}\right)$ ) are called ultradifferentiable functions of Beurling (resp. Roumieu) type $\omega$. We write $\mathcal{E}_{*}\left(\mathbb{R}^{N}\right)$ where $*$ can be either $(\omega)$ or $\{\omega\}$. For a compact set $K$ in $\mathbb{R}^{N}$ we put

$$
\mathcal{D}_{*}(K):=\left\{f \in \mathcal{E}_{*}\left(\mathbb{R}^{N}\right): \operatorname{supp}(f) \subset K\right\}
$$

endowed with the induced topology. For a fundamental sequence $\left(K_{j}\right)_{j \in \mathbb{N}}$ of compact subsets of $\mathbb{R}^{N}$ we let

$$
\mathcal{D}_{*}\left(\mathbb{R}^{N}\right):=\operatorname{ind}_{j \rightarrow} \mathcal{D}_{*}\left(K_{j}\right) .
$$

The elements of $\mathcal{D}_{(\omega)}^{\prime}\left(\mathbb{R}^{N}\right)$ (resp. $\mathcal{D}_{\{\omega\}}^{\prime}\left(\mathbb{R}^{N}\right)$ ) are called ultradistributions of Beurling (resp. Roumieu) type.

Examples of weight functions with and without property $(\epsilon)$ can be found in [10] where it is shown that given a sequence $\left(M_{p}\right)_{p \in \mathbb{N}_{0}}$ satisfying the conditions $(M 1),(M 2)$ and $(M 3)$ of Komatsu [8], there is a concave strong weight $\kappa$ such that $\mathcal{E}_{\left(M_{j}\right)}\left(\mathbb{R}^{N}\right)=\mathcal{E}_{(\kappa)}\left(\mathbb{R}^{N}\right)$, where $\mathcal{E}_{\left(M_{j}\right)}\left(\mathbb{R}^{N}\right)$ is the set of all functions $f \in \mathcal{C}^{\infty}\left(\mathbb{R}^{N}\right)$ such that $\sup _{\alpha \in \mathbb{N}_{0}^{N}} \sup _{x \in K} \frac{\left|f^{(\alpha)}(x)\right|}{h^{|\alpha|} M_{|\alpha|}}<\infty$ for each $h>0$ and each compact subset $K$ of $\mathbb{R}^{N}$. Therefore our results below properly extend the case of almost periodic ultradistributions in [5]. 


\section{Preliminary Results}

Definition 2.1. For a weight function $\omega$, we denote

$$
\mathcal{D}_{L^{1}, \omega, \lambda}:=\left\{f \in \mathcal{D}_{L^{1}}\left(\mathbb{R}^{N}\right):|f|_{\lambda}:=\sup _{\alpha \in \mathbb{N}_{0}^{N}}\left\|f^{(\alpha)}\right\|_{1} \exp \left(-\lambda \varphi^{*}\left(\frac{|\alpha|}{\lambda}\right)\right)<\infty\right\} .
$$

We define $\mathcal{D}_{L^{1},(\omega)}\left(\mathbb{R}^{N}\right):=\operatorname{proj}_{\leftarrow \lambda} \mathcal{D}_{L^{1}, \omega, \lambda}$ and $\mathcal{D}_{L^{1},\{\omega\}}\left(\mathbb{R}^{N}\right):=\operatorname{ind}_{\lambda \rightarrow} \mathcal{D}_{L^{1}, \omega, \lambda}$.

Then $\mathcal{D}_{L^{1},(\omega)}\left(\mathbb{R}^{N}\right)$ is a Fréchet space and $\mathcal{D}_{L^{1},\{\omega\}}\left(\mathbb{R}^{N}\right)$ is an (LB)-space. As usual, we write $\mathcal{D}_{L^{1}, *}\left(\mathbb{R}^{N}\right)$ to denote $\mathcal{D}_{L^{1},(\omega)}\left(\mathbb{R}^{N}\right)$ or $\mathcal{D}_{L^{1},\{\omega\}}\left(\mathbb{R}^{N}\right)$.

The space $\mathcal{D}_{*}\left(\mathbb{R}^{N}\right)$ is dense in $\mathcal{D}_{L^{1}, *}\left(\mathbb{R}^{N}\right)$ and the inclusion $\mathcal{D}_{*}\left(\mathbb{R}^{N}\right) \subset \mathcal{D}_{L^{1}, *}\left(\mathbb{R}^{N}\right)$ is continuous.

In what follows we will always consider $\mathcal{D}_{L^{1}, *}^{\prime}\left(\mathbb{R}^{N}\right)$ endowed with the strong topology $\beta\left(\mathcal{D}_{L^{1}, *}^{\prime}\left(\mathbb{R}^{N}\right), \mathcal{D}_{L^{1}, *}\left(\mathbb{R}^{N}\right)\right)$.

Proposition 2.2. $\mathcal{D}_{L^{1}, *}\left(\mathbb{R}^{N}\right)$ is continuously included in $\mathcal{E}_{*}\left(\mathbb{R}^{N}\right)$.

Proof. We fix an open subset $\Omega \subset \mathbb{R}^{N}$ and a compact set $K \subset \Omega$ and we apply Sobolev's lemma [11, 3.5.12] to find a constant $C>0$ such that

$$
\sup _{x \in K}|f(x)| \leq C \sup _{|\beta| \leq N+1} \int_{\Omega}\left|f^{(\beta)}(t)\right| d t \quad \forall f \in \mathcal{C}^{\infty}(\Omega) .
$$

For every $\lambda>0, \alpha \in \mathbb{N}_{0}^{N}$, and each $f \in \mathcal{D}_{L^{1}, \omega, \lambda}\left(\mathbb{R}^{N}\right)$ we denote by

$$
|f|_{\Omega, \lambda}:=\sup _{\alpha \in \mathbb{N}_{0}^{N}}\left(\int_{\Omega}\left|f^{(\alpha)}(t)\right| d t\right) \exp \left(-\lambda \varphi^{*}\left(\frac{|\alpha|}{\lambda}\right)\right) .
$$

Then $\sup _{x \in K}\left|f^{(\alpha)}(x)\right| \leq C|f|_{\Omega, \lambda} \exp \left(\lambda \varphi^{*}\left(\frac{|\alpha|+N+1}{\lambda}\right)\right)$ for every $f \in \mathcal{C}^{\infty}(\Omega)$. It follows from the convexity of $\varphi^{*}$ that there is $D>0$ with

$$
\exp \left(\lambda \varphi^{*}\left(\frac{|\alpha|+N+1}{\lambda}\right)\right) \leq D \exp \left(\frac{\lambda}{2^{N+1}} \varphi^{*}\left(\frac{2^{N+1}}{\lambda}|\alpha|\right)\right) \quad \forall \alpha \in \mathbb{N}_{0}^{N}
$$

from where we conclude that $\|f\|_{K, \frac{\lambda}{2^{N+1}}} \leq C D|f|_{\Omega, \lambda}$.

Let $G \in \mathcal{H}\left(\mathbb{C}^{N}\right)$ be an entire function such that $\log |G(z)|=\mathcal{O}(\omega(|z|))$ (respect. $\log |G(z)|=o(\omega(|z|)))$ as $|z|$ tends to infinity. Then

$$
T_{G}(\varphi):=\sum_{\alpha \in \mathbb{N}_{0}^{N}}(-i)^{|\alpha|} \frac{G^{(\alpha)}(0)}{\alpha !} \varphi^{(\alpha)}(0)
$$

defines an ultradistribution $T_{G} \in \mathcal{E}_{(\omega)}^{\prime}\left(\mathbb{R}^{N}\right)$ (respect. $T_{G} \in \mathcal{E}_{\{\omega\}}^{\prime}\left(\mathbb{R}^{N}\right)$ ). The operator

$$
G(D): \mathcal{D}_{*}^{\prime}\left(\mathbb{R}^{N}\right) \rightarrow \mathcal{D}_{*}^{\prime}\left(\mathbb{R}^{N}\right), \quad G(D) \nu:=T_{G} * \nu
$$

is called an ultradifferential operator of class $*$. We note that, for every $f \in \mathcal{E}_{*}\left(\mathbb{R}^{N}\right)$,

$$
(G(D) f)(x)=\sum_{\alpha \in \mathbb{N}_{0}^{N}}(i)^{|\alpha|} \frac{G^{(\alpha)}(0)}{\alpha !} f^{(\alpha)}(x) .
$$

Lemma 2.3 (4, 1.4]). There exists $L \geq 1$ such that

$$
L \varphi^{*}\left(\frac{t}{L}\right)+t \leq \varphi^{*}(t)+L \quad \forall t \geq 0
$$


Proposition 2.4. Let $G(D)$ be an ultradifferential operator of class $*$. Then

$$
G(D): \mathcal{D}_{L^{1}, *}\left(\mathbb{R}^{N}\right) \longrightarrow \mathcal{D}_{L^{1}, *}\left(\mathbb{R}^{N}\right)
$$

is a continuous linear mapping.

Proof. We first consider the Beurling case $*=(\omega)$. Let $f \in \mathcal{D}_{L^{1},(\omega)}\left(\mathbb{R}^{N}\right)$ and $m \in \mathbb{N}$. There is $k>0$ such that

$$
\log |G(z)| \leq k\left(1+\omega\left(\frac{|z|}{\sqrt{N}}\right)\right) \quad \forall z \in \mathbb{C}^{N} .
$$

Now we take $h \geq \max (k L, m)$, where $L$ is as in Lemma 2.3. Since $\frac{\varphi^{*}(t)}{t}$ is increasing on $] 0,+\infty\left[\right.$ we get, for every $\alpha \in \mathbb{N}_{0}^{N}$,

$$
|\alpha|+h \varphi^{*}\left(\frac{|\alpha|}{h}\right) \leq|\alpha|+k L \varphi^{*}\left(\frac{|\alpha|}{k L}\right) \leq k \varphi^{*}\left(\frac{|\alpha|}{k}\right)+k L .
$$

We put $\rho=\mathrm{e}^{r}, r>0$, to obtain, after applying the Cauchy inequalities and the convexity of $\varphi^{*}$,

$$
\frac{\left|G^{(\alpha)}(0)\right|}{\alpha !} \int\left|f^{(\alpha+\beta)}(t)\right| d t \leq|f|_{2 h} \exp \left(h \varphi^{*}\left(\frac{|\alpha|}{h}\right)+h \varphi^{*}\left(\frac{|\beta|}{h}\right)\right) \mathrm{e}^{k} \mathrm{e}^{k \varphi(r)-|\alpha| r}
$$

Since $\inf _{r>0} \mathrm{e}^{k \varphi(r)-|\alpha| r}=\exp \left(-k \varphi^{*}\left(\frac{|\alpha|}{k}\right)\right)$ we get from (2.1) and (2.2)

$$
\begin{aligned}
& \frac{\left|G^{(\alpha)}(0)\right|}{\alpha !}\left(\int\left|f^{(\alpha+\beta)}(t)\right| d t\right) \exp \left(-h \varphi^{*}\left(\frac{|\beta|}{h}\right)\right) \\
& \leq|f|_{2 h} \mathrm{e}^{k} \exp \left(-k \varphi^{*}\left(\frac{|\alpha|}{k}\right)+h \varphi^{*}\left(\frac{|\alpha|}{h}\right)\right) \\
& \leq|f|_{2 h} \mathrm{e}^{k} \mathrm{e}^{k L-|\alpha|}
\end{aligned}
$$

and consequently

$$
\sup _{\beta \in \mathbb{N}_{0}^{N}} \sum_{\alpha} \frac{\left|G^{(\alpha)}(0)\right|}{\alpha !}\left(\int\left|f^{(\alpha+\beta)}(t)\right| d t\right) \exp \left(-m \varphi^{*}\left(\frac{|\beta|}{m}\right)\right) \leq C|f|_{2 h}
$$

for some constant $C>0$, which yields $|G(D) f|_{m} \leq C|f|_{2 h}$.

We now discuss the Roumieu case. Let $f \in \mathcal{D}_{L^{1},\{\omega\}}\left(\mathbb{R}^{N}\right)$ and $\lambda>0$ such that $|f|_{2 \lambda}<+\infty$. We take $k:=\frac{\lambda}{L}$ and $A>0$ such that $\log |G(z)| \leq k \omega\left(\frac{|z|}{\sqrt{N}}\right)+A$ for each $z \in \mathbb{C}^{N}$. We proceed as in the Beurling case with $m:=\lambda$ and $h:=$ $\lambda(\geq \max (k L, m))$ to get a constant $C>0$ (independent of $f$ ) satisfying

$$
\sup _{\beta \in \mathbb{N}_{0}^{N}} \sum_{\alpha} \frac{\left|G^{(\alpha)}(0)\right|}{\alpha !}\left(\int\left|f^{(\alpha+\beta)}(t)\right| d t\right) \exp \left(-\lambda \varphi^{*}\left(\frac{|\beta|}{\lambda}\right)\right) \leq C|f|_{2 \lambda} .
$$

The strong non-quasianalyticity condition allowed Cioranescu [5] to find ultradifferential operators admitting a fundamental solution with analytic extension to $\mathbb{C} \backslash\{0\}$. We now show how an argument of Langenbruch [9] can be adapted to 
get ultradifferential operators with a fundamental solution which is real-analytic on $\mathbb{R}^{N} \backslash\{0\}$ and satisfying good enough estimates around the origin. This will permit us to construct "good" parametrices even in the case where the weight does not satisfy the strong non-quasianalytic condition.

Proposition 2.5. Let $\omega$ be a weight function. For every $m \in \mathbb{N}$, there are an ultradifferential operator $G(D)$ of class $(\omega)$ and a function $F \in C^{\infty}\left(\mathbb{R}^{N}\right)$ such that

$$
\sup _{x \in \mathbb{R}^{N}} \sup _{\alpha \in \mathbb{N}_{0}^{N}}\left|F^{(\alpha)}(x)\right| \exp \left(-m \varphi^{*}\left(\frac{|\alpha|}{m}\right)\right)<+\infty
$$

$F$ is real-analytic on $\mathbb{R}^{N} \backslash\{0\}$ and $G(D) F=\delta$.

Proof. Let $L \geq 1$ be a constant satisfying $\omega(t) \leq L\left(1+\omega\left(\frac{t}{\mathrm{e}}\right)\right)$ for every $t \geq 0$. We apply [9, 1.3] as in the proof of [9, 1.4] to the weight function $\sigma(t):=(1+m L) \omega\left(\frac{t}{\mathrm{e}}\right)$ to find an even entire function $f \in \mathcal{H}\left(\mathbb{C}^{N}\right)$ and constants $C_{i}>0$ such that

$$
\log |f(z)| \leq C_{1} \sigma(|z|)+C_{1} \quad \text { for each } z \in \mathbb{C}^{N}
$$

and

$$
\log |f(z)| \geq C_{2} \sigma(|z|) \quad \text { for every } z \in \mathbb{C}^{N}
$$

satisfying $|\operatorname{Imz}| \leq C_{3}(|\operatorname{Re} z|+1)$.

We take $k \in \mathbb{N}$ such that $k C_{2}>1$ and we consider the ultradifferential operator $G(D)$ of class $(\omega)$ defined by the entire function $G(z):=(f(z))^{k}$. We define

$$
F(x):=\frac{1}{(2 \pi)^{N}} \int_{\mathbb{R}^{N}} \frac{\mathrm{e}^{i\langle x, t\rangle}}{G(t)} d t, \quad x \in \mathbb{R}^{N} .
$$

The function $F$ is in $\mathcal{C}^{\infty}\left(\mathbb{R}^{N}\right)$ and $\left|F^{(\alpha)}(x)\right| \leq \frac{1}{(2 \pi)^{N}} \int_{\mathbb{R}^{N}} \frac{|t|^{|\alpha|}}{\mathrm{e}^{\sigma(|t|)}} d t$. Since $\log t=$ $o(\omega(t))$ we get, for some constant $C>0$,

$$
\begin{aligned}
\left|F^{(\alpha)}(x)\right| & \leq \frac{1}{(2 \pi)^{N}} \int_{\mathbb{R}^{N}}|t|^{|\alpha|} \exp \left(-(1+m L)\left(\frac{\omega(|t|)}{L}-1\right)\right) d t \\
& \leq C \exp \left(\sup _{|t| \geq 1}(|\alpha| \log |t|-m \omega(|t|))\right) \\
& =C \exp \left(m \varphi^{*}\left(\frac{|\alpha|}{m}\right)\right)
\end{aligned}
$$

and consequently $\sup _{x \in \mathbb{R}^{N}} \sup _{\alpha \in \mathbb{N}_{0}^{N}}\left|F^{(\alpha)}(x)\right| \exp \left(-m \varphi^{*}\left(\frac{|\alpha|}{m}\right)\right)<+\infty$.

The same argument of Langenbruch [9, 1.4] now shows that $F$ is real-analytic on $\mathbb{R}^{N} \backslash\{0\}$ and $G(D) F=\delta$.

We denote by $B_{\varepsilon}$ the closed ball with radius $\varepsilon>0$ centered at the origin.

Corollary 2.6. For every $m \in \mathbb{N}$ and $\varepsilon>0$, there exist an ultradifferential operator $G(D)$ of class $(\omega)$ and two functions $\chi \in \mathcal{D}_{(\omega)}\left(\mathbb{R}^{N}\right)$ and $\Gamma \in \mathcal{D}\left(B_{\varepsilon}\right) \cap \mathcal{E}_{\omega}\left(B_{\varepsilon}, m\right)$ such that $G(D) \Gamma+\chi=\delta$. 
Proof. Let $n$ be a natural number, $n \geq L m$, with $L$ the constant of Lemma 2.3. We choose $\psi \in \mathcal{D}_{(\omega)}\left(B_{\varepsilon}\right)$ such that $\psi=1$ near 0 and we set $\Gamma:=\psi F$ and $\chi:=$ $G(D)(F-\psi F)$ with $G(D)$ and $F$ from Proposition 2.5 (but replacing $m$ by $n$ ). Compare with the proof of [4, 4.4].

\section{Bounded Ultradistributions}

The aim of this section is to characterize the elements of $\mathcal{D}_{L^{1}, *}^{\prime}\left(\mathbb{R}^{N}\right)$, known as bounded ultradistributions. Once we have established in Proposition 2.5 the existence of ultradifferential operators with "very good" fundamental solutions, the proof of the main theorem for the Beurling case follows the steps of [ $[5]$. The following result will permit us to reduce the study of bounded ultradistributions of Roumieu type to the Beurling case. It should by compared with [4, 7.6].

Proposition 3.1. Let $T \in \mathcal{D}_{\{\omega\}}^{\prime}\left(\mathbb{R}^{N}\right)$. Then the following conditions are equivalent:

(1) $T \in \mathcal{D}_{L^{1},\{\omega\}}^{\prime}\left(\mathbb{R}^{N}\right)$,

(2) there is a weight function $\sigma$ with $\sigma=o(\omega)$ such that $T \in \mathcal{D}_{L^{1},(\sigma)}^{\prime}\left(\mathbb{R}^{N}\right)$.

Proof. $(2) \Rightarrow(1)$ is easy. We now prove $(1) \Rightarrow(2)$. Let function $\phi \in \mathcal{D}_{(\omega)}\left(\mathbb{R}^{N}\right)$ be such that $\sum_{\nu \in \mathbf{Z}^{N}} \phi(x-\nu)=1$ for all $x \in \mathbb{R}^{N}$. We define $S_{\nu} f:=\langle T, f(x-\nu) \phi(x-\nu)\rangle$ for $f \in \mathcal{D}_{\{\omega\}}\left(\mathbb{R}^{N}\right)$ and $\nu \in \mathbf{Z}^{\mathrm{N}}$. Then $S_{\nu} \in \mathcal{D}_{\{\omega\}}^{\prime}\left(\mathbb{R}^{N}\right)$ and $\operatorname{supp} S_{\nu} \subseteq \operatorname{supp} \phi$ $\forall \nu \in \mathbf{Z}^{\mathrm{N}}$.

We claim that $\left(S_{\nu}: \nu \in \mathbf{Z}^{\mathrm{N}}\right)$ is equicontinuous in $\mathcal{E}_{\{\omega\}}^{\prime}\left(\mathbb{R}^{N}\right)$. In fact, since $\mathcal{E}_{\{\omega\}}\left(\mathbb{R}^{N}\right)$ is a projective limit of (DFN)-spaces (hence barrelled) [4, 4.5,3.6] and $\operatorname{supp} S_{\nu} \subset \operatorname{supp} \phi \forall \nu \in \mathbf{Z}^{\mathrm{N}}$, it suffices to show that $\left\{S_{\nu} f: \nu \in \mathrm{Z}^{\mathbf{N}}\right\}$ is a bounded set for every $f \in \mathcal{D}_{\{\omega\}}\left(\mathbb{R}^{N}\right)$. To this aim we fix $f \in \mathcal{D}_{\{\omega\}}\left(\mathbb{R}^{N}\right)$ and we observe that $\left(f_{\nu} \phi_{\nu}: \nu \in \mathrm{Z}^{\mathbf{N}}\right\}$ is a bounded set in $\mathcal{D}_{L^{1},\{\omega\}}\left(\mathbb{R}^{N}\right)$, where we denote by $\varphi_{h}$ the function $\varphi_{h}(t):=\varphi(t+h)$. Since $T \in \mathcal{D}_{L^{1},\{\omega\}}^{\prime}\left(\mathbb{R}^{N}\right)$ we conclude that $\left\{S_{\nu} f: \nu \in\right.$ $\left.Z^{N}\right\}$ is bounded and the claim is proved.

We can apply [4, 7.6] to find a weight function $\sigma$ such that $\sigma=o(\omega)$ and $\left\{S_{\nu}: \nu \in \mathrm{Z}^{\mathbf{N}}\right\}$ is an equicontinuous set in $\mathcal{E}_{(\sigma)}^{\prime}\left(\mathbb{R}^{N}\right)$. We now define

$$
\left\langle\tau_{-\nu} S_{\nu}, f\right\rangle:=\left\langle S_{\nu}, f(x+\nu)\right\rangle, \quad f \in \mathcal{D}_{L^{1},(\sigma)}\left(\mathbb{R}^{N}\right) .
$$

Our next aim is to show that $\sum_{\nu \in Z^{\mathbf{N}}} \tau_{-\nu} S_{\nu}$ defines an element of $\mathcal{D}_{L^{1},(\sigma)}^{\prime}\left(\mathbb{R}^{N}\right)$. To do this we note that there are constants $C>0, k \in \mathbb{N}$ such that for every $f \in \mathcal{D}_{L^{1},(\sigma)}\left(\mathbb{R}^{N}\right)$ and for each $\nu \in \mathrm{Z}^{\mathbf{N}}$ we have

$$
\left|\left\langle\tau_{-\nu} S_{\nu}, f\right\rangle\right| \leq C \sup _{\alpha \in \mathbb{N}_{o}^{N}} \sup _{\|x\|_{\infty} \leq k}\left|f^{(\alpha)}(x+\nu)\right| \exp \left(-k \varphi_{\sigma}^{*}\left(\frac{|\alpha|}{k}\right)\right) .
$$

We put $Q=\left\{x:\|x\|_{\infty} \leq k+1\right\}$ and we proceed as in the proof of Proposition 2.2 to get, for some constant $C>0$ which is not the same at each ocurrence and some 
$m>0$,

$$
\begin{aligned}
& \sum_{\nu \in Z^{\mathbb{N}}}\left|\left\langle\tau_{-\nu} S_{\nu}, f\right\rangle\right| \\
& \leq C \sum_{\alpha \in \mathbb{N}_{0}^{N}} \sum_{\nu \in Z^{\mathbb{N}}}\left(\int_{Q+\nu}\left|f^{(\alpha)}\right|\right) \exp \left(-k \varphi_{\sigma}^{*}\left(\frac{|\alpha|}{k}\right)\right) \\
& \leq C \sum_{\alpha \in \mathbb{N}_{0}^{N}}\left\|f^{(\alpha)}\right\|_{1} \exp \left(-k \varphi_{\sigma}^{*}\left(\frac{|\alpha|}{k}\right)\right) \\
& \leq C \sup _{\alpha \in \mathbb{N}_{0}^{N}}\left\|f^{(\alpha)}\right\|_{1} \exp \left(-m \varphi_{\sigma}^{*}\left(\frac{|\alpha|}{m}\right)\right) .
\end{aligned}
$$

Moreover, for every $f \in \mathcal{D}_{\{\omega\}}\left(\mathbb{R}^{N}\right)$ since $\sum_{\nu \in Z^{\mathbb{N}}} f(x) \phi(x-\nu)=f(x)$, we obtain

$$
\sum_{\nu \in \mathrm{Z}^{\mathbf{N}}}\left\langle\tau_{-\nu} S_{\nu}, f\right\rangle=\sum_{\nu \in \mathrm{Z}^{\mathbf{N}}}\left\langle S_{\nu}, f(x+\nu)\right\rangle=\sum_{\nu \in \mathrm{Z}^{\mathbf{N}}}\langle T, f(x) \phi(x-\nu)\rangle=\langle T, f\rangle,
$$

showing that $\sum_{\nu \in \mathrm{Z}^{\mathrm{N}}} \tau_{-\nu} S_{\nu}$ is an extension of $T$ to $\mathcal{D}_{L^{1},(\sigma)}^{\prime}\left(\mathbb{R}^{N}\right)$.

From now on we denote by $\mathcal{C}_{b}\left(\mathbb{R}^{N}\right)$ the Banach space of bounded and continuous functions on $\mathbb{R}^{N}$.

Theorem 3.2. Let $\omega$ be a weight function and let $T \in \mathcal{D}_{*}^{\prime}\left(\mathbb{R}^{N}\right)$. Then, the following conditions are equivalent:

(1) $T \in \mathcal{D}_{L^{1}, *}^{\prime}\left(\mathbb{R}^{N}\right)$,

(2) there exist an ultradifferential operator $G(D)$ of class $*$ and two bounded and continuous functions $f, g$ such that $T=G(D) f+g$,

(3) $T * \varphi \in \mathcal{C}_{b}\left(\mathbb{R}^{N}\right)$ for every $\varphi \in \mathcal{D}_{*}\left(\mathbb{R}^{N}\right)$.

Proof. The Beurling case $*=(\omega)$ follows as in Cioranescu [5, Theorem 1] after replacing [5, Lemma 2] by Corollary 2.6.

We sketch the argument.

$(1) \Rightarrow(3)$. We observe that for each $\varphi \in \mathcal{D}_{(\omega)}\left(\mathbb{R}^{N}\right)$ the set of translates $\left\{\varphi_{h}\right.$ : $\left.h \in \mathbb{R}^{N}\right\}$ is bounded in $\mathcal{D}_{L^{1},(\omega)}\left(\mathbb{R}^{N}\right)$. Now the continuity of $T$ gives the conclusion.

$(2) \Rightarrow(1)$. For every $\varphi \in \mathcal{D}_{L^{1},(\omega)}\left(\mathbb{R}^{N}\right),\langle T, \varphi\rangle=\int f G(-D) \varphi+\int g \varphi$. Since $G(-D)$ is a continuous linear operator on $\mathcal{D}_{L^{1}, *}\left(\mathbb{R}^{N}\right)$, we conclude that $T \in \mathcal{D}_{L^{1},(\omega)}^{\prime}\left(\mathbb{R}^{N}\right)$.

(3) $\Rightarrow(2)$. We note that for all compact subsets $K$ of $\mathbb{R}^{N}$ there is $m \in \mathbb{N}$ such that $T * \varphi \in C_{b}\left(\mathbb{R}^{N}\right)$ for each $\varphi \in \mathcal{E}_{\omega}(K, m) \cap D(K)$. Now take $G(D), \chi$ and $\Gamma$ as in Corollary 2.6 and put $f:=T * \Gamma, g:=T * \chi$.

We now consider the Roumieu case $*=\{\omega\}$.

$(1) \Rightarrow(2)$. There exists a weight function $\sigma$ such that $\sigma=o(\omega)$ and $T \in$ $\mathcal{D}_{L^{1},(\sigma)}^{\prime}\left(\mathbb{R}^{N}\right)$. Consequently there exist an ultradifferential operator $G(D)$ of class $(\sigma)$ and two functions $f, g \in \mathcal{C}_{b}\left(\mathbb{R}^{N}\right)$ satisfying $T=G(D) f+g$ on $\mathcal{D}_{(\sigma)}^{\prime}\left(\mathbb{R}^{N}\right)$. Since $\sigma=o(\omega)$ it follows that $G(D)$ is an ultradifferential operator of class $\{\omega\}$ and $T=G(D) f+g$ on $\mathcal{D}_{\{\omega\}}^{\prime}\left(\mathbb{R}^{N}\right)$.

$(2) \Rightarrow(1)$. Since every bounded and continuous function is a bounded ultradistribution and $G(D)$ is a continuous linear operator on $\mathcal{D}_{L^{1},\{\omega\}}^{\prime}\left(\mathbb{R}^{N}\right)$ we conclude that $T$ is a bounded ultradistribution.

$(1) \Rightarrow(3)$. The proof is as in the Beurling case. 
(3) $\Rightarrow(1)$. We define $\left(S_{\nu}: \nu \in \mathrm{Z}^{\mathbf{N}}\right)$ as in the proof of Proposition 3.1. For every $f \in \mathcal{D}_{\{\omega\}}\left(\mathbb{R}^{N}\right)$ we have $\left\langle S_{\nu}, f\right\rangle=(T *(\check{f} \check{\phi}))(\nu)$. Consequently $\left(S_{\nu}: \nu \in \mathrm{Z}^{\mathbf{N}}\right)$ is a bounded set in $\mathcal{E}_{\{\omega\}}^{\prime}\left(\mathbb{R}^{N}\right)$ and we proceed as in Proposition 3.1 to find a weight $\sigma$ such that $\sigma=o(\omega)$ and $\sum_{\nu \in \mathrm{Z}^{N}} \tau_{-\nu} S_{\nu}$ belongs to $\mathcal{D}_{L^{1},(\sigma)}^{\prime}\left(\mathbb{R}^{N}\right)$.

For a distribution $T \in \mathcal{D}_{*}^{\prime}\left(\mathbb{R}^{N}\right)$ and $h \in \mathbb{R}^{N}, T_{h}$ is defined by

$$
\left\langle T_{h}, \varphi\right\rangle:=T\left(\varphi_{-h}\right), \quad \varphi_{-h}(t):=\varphi(t-h) .
$$

The condition (3) in the theorem above is equivalent to being $\left\{T_{h}: h \in \mathbb{R}^{N}\right\}$, a bounded set in $\mathcal{D}_{*}^{\prime}\left(\mathbb{R}^{N}\right)$.

The arguments of Theorem 3.2 also show the following result, which will be used to extend the Bochner-Féjer summation method to almost periodic ultradistributions.

Corollary 3.3. Let $\left(T_{j}\right)$ be a sequence of bounded ultradistributions $T_{j} \in \mathcal{D}_{L^{1}, *}^{\prime}\left(\mathbb{R}^{N}\right)$. Then the following conditions are equivalent:

(1) The sequence $\left(T_{j}\right)$ converges to zero in $\mathcal{D}_{L^{1}, *}^{\prime}\left(\mathbb{R}^{N}\right)$,

(2) $\left(T_{j} * \varphi\right)$ converges to zero in $\mathcal{C}_{b}\left(\mathbb{R}^{N}\right)$ for every $\varphi \in \mathcal{D}_{*}\left(\mathbb{R}^{N}\right)$,

(3) there exist an ultradifferential operator $G(D)$ of class $*$ and two null sequences $\left(f_{j}\right)$ and $\left(g_{j}\right)$ in $\mathcal{C}_{b}\left(\mathbb{R}^{N}\right)$ such that $T_{j}=G(D) f_{j}+g_{j}$.

\section{Almost periodic ultradistributions}

We recall that a funcion $f \in \mathcal{C}_{b}\left(\mathbb{R}^{N}\right)$ is said to be almost periodic if $f$ is the uniform limit of a sequence of trigonometric polynomials. This is equivalent to being the set of translates $\left\{f_{h}: h \in \mathbb{R}^{N}\right\}$, a relatively compact set in $\mathcal{C}_{b}\left(\mathbb{R}^{N}\right)([7])$. The set of the almost periodic functions will be denoted by $\mathcal{C}_{a p}\left(\mathbb{R}^{N}\right)$.

Definition 4.1. We say that $T \in \mathcal{D}_{L^{1}, *}^{\prime}\left(\mathbb{R}^{N}\right)$ is almost periodic if $T$ is the limit in $\mathcal{D}_{L^{1}, *}^{\prime}\left(\mathbb{R}^{N}\right)$ of a sequence of trigonometric polynomials.

Theorem 4.2. For $T \in \mathcal{D}_{L^{1}, *}^{\prime}\left(\mathbb{R}^{N}\right)$, the following conditions are equivalent:

(1) $T$ is almost periodic,

(2) there exist an ultradifferential operator $G(D)$ of class $*$ and two almost periodic continuous functions $f, g \in \mathcal{C}_{a p}\left(\mathbb{R}^{N}\right)$ such that $T=G(D) f+g$,

(3) $T * \varphi \in \mathcal{C}_{a p}\left(\mathbb{R}^{N}\right)$ for every $\varphi \in \mathcal{D}_{*}\left(\mathbb{R}^{N}\right)$,

(4) the set of translates $\left(T_{h}: h \in \mathbb{R}^{N}\right)$ is relatively compact in $\mathcal{D}_{L^{1}, *}^{\prime}\left(\mathbb{R}^{N}\right)$.

In the Roumieu case, $*=\{\omega\}$, the above conditions are equivalent to:

(5) there exists a weight function $\sigma$ with $\sigma=o(\omega)$ such that $T$ is almost periodic in $\mathcal{D}_{L^{1},(\sigma)}^{\prime}\left(\mathbb{R}^{N}\right)$.

Proof. (2) implies (4) follows from the fact that $\left(f_{h}: h \in \mathbb{R}^{N}\right)$ and $\left(g_{h}: h \in \mathbb{R}^{N}\right)$ are relatively compact subsets of $C_{b}\left(\mathbb{R}^{N}\right)$ and $G(D): \mathcal{D}_{L^{1}, *}^{\prime}\left(\mathbb{R}^{N}\right) \longrightarrow \mathcal{D}_{L^{1}, *}^{\prime}\left(\mathbb{R}^{N}\right)$ is a continuous and linear operator. To prove that (4) implies (3) we fix $\varphi \in \mathcal{D}_{*}\left(\mathbb{R}^{N}\right)$ and we observe that the map

$$
\mathcal{D}_{L^{1}, *}^{\prime}\left(\mathbb{R}^{N}\right) \longrightarrow C_{b}\left(\mathbb{R}^{N}\right), \quad R \longmapsto R * \varphi
$$

is continuous. Therefore $\left\{(T * \varphi)_{h}: h \in \mathbb{R}^{N}\right\}=\left\{T_{h} * \varphi: h \in \mathbb{R}^{N}\right\}$ is relatively compact in $C_{b}\left(\mathbb{R}^{N}\right)$ and hence $T * \varphi$ is an almost periodic function. 
To prove the rest of the equivalences we treat the Beurling and the Roumieu cases separately. In the Beurling case, the equivalence between conditions (1), (2) and (3) can be obtained as in [5. Theorem 2].

We now consider the Roumieu case $*=\{\omega\}$.

$(3) \Rightarrow(5)$. We apply Proposition 3.1 to find a weight $\sigma$ with $\sigma=o(\omega)$ such that $T \in \mathcal{D}_{L^{1},(\sigma)}^{\prime}\left(\mathbb{R}^{N}\right)$. This means that the set of translates of $T,\left\{T_{h}: h \in \mathbb{R}^{N}\right\}$, is bounded in $\mathcal{D}_{(\sigma)}^{\prime}\left(\mathbb{R}^{N}\right)$ and consequently the map $\mathcal{D}_{(\sigma)}\left(\mathbb{R}^{N}\right) \rightarrow C_{b}\left(\mathbb{R}^{N}\right)$ given by $\varphi \rightarrow T * \varphi$ is continuous. Since $\mathcal{D}_{\{\omega\}}\left(\mathbb{R}^{N}\right)$ is dense in $\mathcal{D}_{(\sigma)}\left(\mathbb{R}^{N}\right)([4,3.9])$ and $T * \psi$ can be uniformly approximated by trigonometric polynomials for each $\psi \in \mathcal{D}_{\{\omega\}}\left(\mathbb{R}^{N}\right)$ we obtain that $T * \varphi$ is an almost periodic function for every $\varphi \in$ $\mathcal{D}_{(\sigma)}\left(\mathbb{R}^{N}\right)$. Consequently $T$ is almost periodic in $\mathcal{D}_{L^{1},(\sigma)}^{\prime}\left(\mathbb{R}^{N}\right)$. Clearly (5) implies all the other conditions, while $(1) \Rightarrow(3)$ and $(2) \Rightarrow(1)$ can be obtained as in the Beurling case.

We recall that the Fourier coefficients of an almost periodic function $f: \mathbb{R} \rightarrow \mathbb{C}$ are defined as $c(\lambda):=M\left(f(x) e^{-i \lambda x}\right), \lambda \in \mathbb{R}$, where $M(g)$ denotes the mean value $M(g):=\lim _{x \rightarrow \infty} \frac{1}{2 x} \int_{-x}^{x} g(t) d t$. It is well known that $c(\lambda)=0$ except for at most a countable set of values of $\lambda$, which is called the spectrum of $f$. Let $f$ be an almost periodic function with spectrum $\Lambda=\left\{\lambda_{n}: n \in \mathbb{N}\right\}$ and we put $c_{n}=c\left(\lambda_{n}\right)$. Then $\sum_{n=1}^{\infty} c_{n} e^{i \lambda_{n} x}$ is the Fourier series of $f$. We refer to [7].

Definition 4.3. Let $S \in \mathcal{D}_{L^{1}, *}^{\prime}(\mathbb{R})$ be an almost periodic ultradistribution. Then the mean value of $S$ is defined as

$$
M(S):=\frac{M(S * \varphi)}{\hat{\varphi}(0)}
$$

where $\varphi$ is a fixed test function $\varphi \in \mathcal{D}_{*}(\mathbb{R})$ such that $\hat{\varphi}(0) \neq 0$.

The above definition does not depend of the fixed function $\varphi$. This easily follows from the fact that $M(f * \varphi)=M(f) \hat{\varphi}(0)$ whenever $f$ is an almost periodic continuous function and $\varphi$ is a test function.

Definition 4.4. The Fourier coefficients of an almost periodic ultradistribution $S \in \mathcal{D}_{L^{1}, *}^{\prime}(\mathbb{R})$ are defined as $C_{S}(\lambda):=M\left(e^{-i \lambda x} S\right), \lambda \in \mathbb{R}$.

Proposition 4.5. (1) Let $S \in \mathcal{D}_{*}^{\prime}(\mathbb{R})$ be an almost periodic ultradistribution. Then, the spectrum of $S, \Lambda_{S}:=\left\{\lambda \in \mathbb{R}: C_{S}(\lambda) \neq 0\right\}$ is a countable set.

(2) Two almost periodic ultradistributions with the same Fourier coefficients are equal.

Proof. We put $v_{\lambda}(x)=e^{-i \lambda x}$ and we fix a test function $\phi \in \mathcal{D}_{*}(\mathbb{R})$ satisfying that $\hat{\phi}(0)=1$. We now take an arbitrary non-null test function $\varphi$ and we observe that $M\left(v_{\lambda}(S * \varphi)\right)=M\left(v_{\lambda} S * v_{\lambda} \varphi\right)=M\left(v_{\lambda} S * \phi\right) \widehat{v_{\lambda} \varphi}(0)=M\left(v_{\lambda} S\right) \hat{\varphi}(\lambda)$. Since the spectrum of the almost periodic function $S * \varphi$ is a countable set and $\hat{\varphi}$ is an entire function it follows that the spectrum of $S$ is countable and the proof of (1) is finished. To prove (2) let us assume that $S_{1}, S_{2} \in \mathcal{D}_{*}^{\prime}(\mathbb{R})$ satisfies that $C_{S_{1}}(\lambda)=C_{S_{2}}(\lambda)$ for every $\lambda \in \mathbb{R}$. Then for $S:=S_{1}-S_{2}$, we have $M\left(v_{\lambda}(S * \varphi)\right)=$ $M\left(v_{\lambda} S\right) \hat{\varphi}(\lambda)=0$ for every test function $\varphi$. We apply [7] 1.19] to get $S * \varphi=0$ for every test function $\varphi$ and consequently $S=0$. 
We now discuss the Bochner-Féjer summation method for ultradistributions.

Theorem 4.6. Let $S \in \mathcal{D}_{L^{1}, *}^{\prime}(\mathbb{R})$ be an almost periodic ultradistribution with spectrum $\Lambda=\left\{\lambda_{k}\right\}$ and Fourier coefficients $a_{k}:=C_{S}\left(\lambda_{k}\right)$. Then, there exists a sequence of trigonometric polynomials

$$
\sigma_{m}(x):=\sum_{k=1}^{n(m)} r_{k, m} a_{k} e^{i \lambda_{k} x}
$$

such that $\left(\sigma_{m}\right)$ converges to $S$ in the strong topology of $\mathcal{D}_{L^{1}, *}^{\prime}(\mathbb{R})$. The numbers $r_{k, m}$ are rational and depend on $\lambda_{k}$ and $m$, but not on $a_{k}$.

Proof. In fact, we apply [7, theorem 1.24] to find a sequence $n(m)$ of natural numbers and rational numbers $r_{k, m}, 1 \leq k \leq n(m)$, such that every almost periodic function $f$ with spectrum contained in $\left\{\lambda_{k}\right\}$ and with Fourier coefficients $c_{k}$ is the uniform limit of the sequence of trigonometric polynomials $\sum_{k=1}^{n(m)} r_{k, m} c_{k} e^{i \lambda_{k} x}$. In order to show that $\left(\sigma_{m}\right)$ converges to $S$ in the strong topology of $\mathcal{D}_{L^{1}, *}^{\prime}(\mathbb{R})$ we fix a test function $\varphi \in \mathcal{D}_{*}(\mathbb{R})$ and we observe that the spectrum of $S * \varphi$ is contained in $\left(\lambda_{k}\right)$, while the Fourier coefficients are given by $M\left(v_{\lambda_{k}}(S * \varphi)\right)=a_{k} \hat{\varphi}\left(\lambda_{k}\right)$. Since $\left(\sigma_{m} * \varphi\right)(x)=\sum_{k=1}^{n(m)} r_{k, m} a_{k} \hat{\varphi}\left(\lambda_{k}\right) e^{i \lambda_{k} x}$ we deduce that $S * \varphi$ is the uniform limit of the sequence of trigonometric polynomials $\sigma_{m} * \varphi$. An application of Corollary 3.3 gives the conclusion.

A similar argument permits us to deduce from [7, 1.22;1.25] the following convergence criteria for the Fourier series of an almost periodic ultradistribution.

Proposition 4.7. Let $T \in \mathcal{D}_{L^{1}, *}^{\prime}(\mathbb{R})$ be an almost periodic ultradistribution with spectrum $\Lambda=\left(\lambda_{k}: k \in \mathbb{N}\right)$. Then the Fourier series of $T$ converges to $T$ in the strong topology of $\mathcal{D}_{L^{1}, *}^{\prime}(\mathbb{R})$ if one of the following two conditions holds:

(1) the Fourier exponents $\lambda_{k}$ are linearly independent over the field of the rational numbers,

(2) there exists a constant $a>1$ such that $\frac{\lambda_{k+1}}{\lambda_{k}} \geq a$ for every $k \in \mathbb{N}$.

\section{REFERENCES}

1. L. Amerio, G. Prouse, Almost periodic functions and functional equations, Van Nostrand, New York (1971). MR 43:819

2. G. Björk, Linear partial differential operators and generalized distributions, Ark. Math. 6 (1965), 351-407. MR 34:3054

3. R. W. Braun, An extension of Komatsu's second structure theorem for ultradistributions, J. Fac. Sci. Tokyo Sec. IA 40 (1993), 411-417. MR 95a:46060

4. R. W. Braun, R. Meise, B. A. Taylor, Ultradifferentiable functions and Fourier analysis, Result. Math. 17 (1990), 206-237. MR 91h:46072

5. I. Cioranescu, The characterization of the almost periodic ultradistributions of Beurling type, Proc. Amer. Math. Soc. 116,1, (1992), 127-134. MR 92k:46063

6. I. Cioranescu, Asymptotically almost periodic distributions, Applicable Analysis 34 (1989), 251-259. MR 97h:46058

7. C. Corduneau, Almost periodic functions, Tracts in Pure Appl. Math. 22, Interscience, New York (1968).

8. H. Komatsu, Ultradistributions I, J. Fac. Sci. Univ. Tokyo Sec. IA Math. 20 (1973), 25-105. MR 47:9277

9. M. Langenbruch, Surjective partial differential operators on spaces of ultradifferentiable functions of Roumieu Type, Results Math. 29 (1996), no.3-4, 254-275. MR 97e:47083

10. R. Meise, B. A. Taylor, Whitney's extension theorem for ultradifferentiable functions of Beurling type, Arkiv für matematik 26 (1988), no.2, 265-287. MR 91h:46074 
11. R. Narasimhan, Analysis on Real and Complex Manifolds, Serie Advanced Studies in Pure Mathematics, Ed. Masson and Cie, Paris (1969). MR 49:11576

12. S. Pilipovic, Characterization of bounded sets in spaces of ultradistributions, Proc. Amer. Math. Soc. 120, 4, (1994), 1191-1206. MR 94f:46048

13. S. Pilipovic, Structural theorems for ultradistribution, Structural aspects of differenciability, Dissertationes Mathematicae 340 (1995), 223-235. MR 96f:46076

14. L. Schwartz, Théorie des distributions, Hermann, Paris (1966). MR 35:730

Departamento de Matemática Aplicada, E.T.S. Arquitectura, Camino de Vera, E46071 Valencia, Spain

E-mail address: cgomezc@mat.upv.es 